\title{
The Reciprocity Principle in Gravitational Interactions
}

\author{
Angel Fierros Palacios \\ Instituto de Investigaciones Eléctricas, División de Energías Alternas, Cuernavaca, México \\ Email: afierros@iie.org.mx
}

Received 4 March 2016; accepted 20 March 2016; published 24 March 2016

Copyright (C) 2016 by author and OALib.

This work is licensed under the Creative Commons Attribution International License (CC BY). http://creativecommons.org/licenses/by/4.0/

(c) (i) Open Access

\section{Abstract}

In this paper it is proposed that the ratio between the apparent radius of any two celestial bodies separated by a very big distance, as seemed each other at a distance, is numerically equal to the ratio of the respective real values of their radius. That relationship is what is called in the paper the Reciprocity Principle. In other words, the apparent size of heavenly bodies, as seemed at a distance, plays a special role in the gravitational interactions. This is so because of some kind of effect over the size due to the very big distances in space. When a couple of celestial bodies interact each other, both of them can be considered as sources of the gravity attraction. In that situation, each body acts upon the other in such a way that it can be considered that there are some degree of reciprocal interaction. In this paper, a formal definition of that reciprocal effect is given.

\section{Keywords}

Escape Velocity, Apparent Size, Reciprocity Principle

Subject Areas: Classical Mechanics

\section{Introduction}

Let us consider two heavenly bodies having masses $m_{1}$ and $m_{2}$, and radius $r_{1}$ and $r_{2}$, respectively, separated by a certain distance, and in gravitational attraction acting along the line joining them. In that situation, the values of their escape velocities due to its apparent size are dependent on their masses, and critically on their apparent radius [1]. When those celestial bodies interact each other, both of them must be considered as sources of gravity force. Then, along that dynamical process, as it was said before, there exist some degree of reciprocity [1]. By definition of escape velocity it has that [2]

$$
v_{o}=\left(\frac{2 G M}{R}\right)^{1 / 2}
$$


where $G$ is the Gravitational Constant, $M$ the mass of the source of the gravity force, and $R$ its radius [1].

\section{The Reciprocity Principle}

When the heavenly bodies before mentioned, are in gravitational interaction, it is easy to see that from Equation (1) the following relationship can be obtained

$$
\left(\frac{v_{1}^{\prime}}{v_{2}^{\prime}}\right)^{2}=\left(\frac{m_{1}}{m_{2}}\right) \cdot\left(\frac{r_{2}^{\prime}}{r_{1}^{\prime}}\right)
$$

where the $r^{\prime}$ are the apparent radius of the bodies.

Let's consider one of the bodies as the source of the gravity attraction; and be that body the one marked with the index 2. Its mass is $m_{2}$, and its apparent radius is $r_{2}^{\prime}$. So that, the equation (1) takes the following form [1].

$$
v_{2}^{\prime}=\left(\frac{2 G m_{2}}{r_{2}^{\prime}}\right)^{1 / 2}
$$

In order to obtain $r_{2}^{\prime}$, and also $v_{2}^{\prime}$ it can be use the following procedure. It is well known from Optics that the ratio of the image size $q$, to the object size $p$ is the Magnification A, that is to say

$$
A=\frac{q}{p}
$$

To obtain the apparent size of that body, it can be used some optical astronomical instrument that has a magnification $A$; in order to get the apparent image, and also the apparent size, given by the following formula

$$
p=\frac{q}{A}
$$

Given that $A$ is a number, $r_{2}^{\prime}$ is equal to $p / 2$. To calculate $v_{2}^{\prime}$ is enough to introduce into Equation (2) the result obtained. However, in that equation it has the unknown quantity $r_{1}^{\prime}$. In order to known its value, it is proposed that:

The ratio between the apparent radius $r_{2}^{\prime} / r_{1}^{\prime}$ keep the same proportion among them as the ratio of the respectives real values of their radius; that is to say

$$
\frac{r_{2}^{\prime}}{r_{1}^{\prime}}=\frac{r_{2}}{r_{1}}
$$

where $r_{1}$ and $r_{2}$ are the values of the real radius. The proposition before given is the Reciprocity Principle.

\section{The Sun-Earth System}

As an illustrative example, let's consider the case of the gravitational interaction between the Sun and our planet. Therefore, Equation (2) becomes

$$
\left(\frac{v_{T}^{\prime}}{v_{\odot}^{\prime}}\right)^{2}=\left(\frac{M_{T}}{M_{\odot}}\right) \cdot\left(\frac{R_{\odot}^{\prime}}{R_{T}^{\prime}}\right)
$$

Then, it has that

$$
\frac{v_{T}^{\prime}}{v_{\odot}^{\prime}}=\left[\left(\frac{M_{T}}{M_{\odot}}\right)\left(\frac{R_{\odot}}{R_{T}}\right)\right]^{1 / 2}
$$

according to relationship (6).

Taking into account the values that appears in the Appendix, it is obtained that

$$
\frac{M_{T}}{M_{\odot}}=2.82 \times 10^{-6}
$$


and

$$
\frac{R_{\odot}}{R_{T}}=1.09 \times 10^{2}
$$

in such a way that in Equation (8) it has that

$$
\left[\left(\frac{M_{T}}{M_{\odot}}\right)\left(\frac{R_{\odot}}{R_{T}}\right)\right]^{1 / 2}=1.753 \times 10^{-2}
$$

Substituting this result into Equation (8) the following value for the Earth's escape velocity due to its apparent size is obtained; that is to say

$$
v_{T}^{\prime}=1.753 \times 10^{-2} v_{\odot}^{\prime}
$$

where $v_{\odot}^{\prime}=13 c$, and $c$ is the speed of light in vacuum [1]. Then

$$
v_{T}^{\prime}=0.228 \mathrm{c}=68,371 \mathrm{~km} \cdot \mathrm{sec}^{-1}
$$

That result means that, due to the smallness of the Earth in comparison to the Sun, its escape velocity $v_{T}^{\prime}$ is orders of magnitude smaller than $v_{\odot}^{\prime}$. Hence, when both of those velocities meets in some point of the space, they pull each other, because they are the carriers of the respective gravity force.

Finally, from Equation (10) it is easy to obtain that

$$
R_{T}^{\prime}=1.59 \times 10^{-4} \mathrm{~km}
$$

Thus,

$$
\frac{R_{\odot}^{\prime}}{R_{T}^{\prime}}=1.09 \times 10^{2}
$$

But, this is the same numerical value given in (10).

\section{The Point of Meet}

It is clear that in the meantime the escape velocity $v_{T}^{\prime}$ travels $68,371 \mathrm{~km}$, the escape velocity $v_{\odot}^{\prime}$ travels a distance equal to $3.9 \times 10^{6} \mathrm{~km}$. Let us suppose that along the whole distance, those velocities maintain its values. Then, let the time transit that takes to $v_{\odot}^{\prime}$ to travel the given distance, be the unit of measure; that is to say

$$
t_{t}=38.19 \text { seconds }
$$

In that time $v_{T}^{\prime}$ travels the distance

$$
x_{T}=2,611,100 \mathrm{~km}
$$

in the meantime $v_{\odot}^{\prime}$ travels the distance

$$
x_{\odot}=147,388,911 \mathrm{~km}
$$

Those results indicate that the point of meet occurs near the Earth

\section{Conclusions}

It is proposed that in Gravitational Interactions, the Reciprocity Principle is valid for any couple of heavenly bodies that interact each other. Also, it is a useful concept, and a powerful tool to calculate the escape velocities and radius due to the apparent size of each body. In other words, its validity can be extended to the whole Universe. On the other hand, it is possible to prove that the ratios $v_{1} / v_{1}^{\prime}$ and $v_{2} / v_{2}^{\prime}$ for two celestial bodies 1 and 2 , respectively, are numerically equal. The same is valid for the ratios $v_{1} / v_{2}$ and $v_{1}^{\prime} / v_{2}^{\prime}$; in such a way that from this point of view; it is always true that

$$
\frac{v_{1}}{v_{1}^{\prime}}=\frac{v_{2}}{v_{2}^{\prime}} ; \text { and } \frac{v_{1}}{v_{2}}=\frac{v_{1}^{\prime}}{v_{2}^{\prime}}
$$


where the prime refers the escape velocity of these bodies due to their apparent size.

In the case of the Sun-Earth System, it has that

$$
\frac{v_{T}}{v_{T}^{\prime}}=1.6 \times 10^{-4} ; \text { and } \frac{v_{\odot}}{v_{\odot}^{\prime}}=1.6 \times 10^{-4}
$$

and also,

$$
\frac{v_{T}}{v_{\odot}}=1.8 \times 10^{-2} ; \text { and } \frac{v_{T}^{\prime}}{v_{\odot}^{\prime}}=1.8 \times 10^{-2}
$$

\section{References}

[1] Fierros Palacios, A. (2015) Gravitation. Scientific Research Publishing, Wuhan. In printing press.

[2] Resnick, R. and Holliday, D. (1966) Physics. John Wiley and Sons Inc., Hoboken.

\section{Appendix}

$$
\begin{aligned}
& M_{T}=5.6 \times 10^{27} \mathrm{gr} \\
& M_{\odot}=1.985 \times 10^{33} \mathrm{gr} \\
& R_{T}=6.378 \times 10^{8} \mathrm{~cm} \\
& R_{\odot}=6.951 \times 10^{10} \mathrm{~cm} \\
& R_{\odot}^{\prime}=1.738 \times 10^{3} \mathrm{~cm}
\end{aligned}
$$

